\title{
Sprites in varying air density: Charge conservation, glowing negative trails and changing velocity
}

\author{
A. Luque ${ }^{1,2}$ and U. Ebert ${ }^{1,3}$ \\ Received 7 December 2009; revised 9 February 2010; accepted 25 February 2010; published 30 March 2010.
}

[1] We study the initial downward propagation of long sprite streamers in naturally varying air density with numerical simulations on adaptively refined grids. We find that the ionization rate and the light emission from the streamer head increase approximately linearly with air density (therefore exponentially with time) while the radius hardly changes, in contradiction with a naive application of scaling laws. Charge conservation creates a negative charge and a consecutively enhanced electric field in the upper section of the streamer channel, while the head carries a growing positive charge. The high electric field in the upper part of the channel generates a secondary ionization wave and a luminous trail. The negative charging explains the emergence of upward, negative streamers from a channel and the attraction of positive heads to a previously formed channel. Finally we show that the vertical gradient in the air density initially accelerates the streamer head and subsequently slows it down, in agreement with recent observations. Citation: Luque, A., and U. Ebert (2010), Sprites in varying air density: Charge conservation, glowing negative trails and changing velocity, Geophys. Res. Lett., 37, L06806, doi:10.1029/2009GL041982.

\section{Introduction}

[2] Sprites are mesospheric filamentary electric discharges created by an intense, normally positive, cloud-to-ground lightning stroke; they propagate over tens of kilometers. The filaments in sprites are streamers [Pasko et al., 1997; Ebert et al., 2006]: thin channels of ionized air that extend due to an enhanced electric field at their tip. This has been confirmed by telescopic imaging by Gerken et al. [2000] and by highspeed recordings that show sprites starting as downwardspropagating bright spots of increasing luminosity [McHarg et al., 2007]; these spots mark the high-field regions of streamers. Other features observed by Stanley et al. [1999], Cummer et al. [2006], McHarg et al. [2007], StenbaekNielsen and McHarg [2008], and Li and Cummer [2009] with increasingly high temporal resolution are: (a) glowing regions in the streamer trail, (b) the emergence of upwardpropagating negative streamers from existing channels of previous streamers, (c) the attraction of streamers towards existing channels, and (d) that sprite streamers initially often accelerate very fast to later gradually slow down.

\footnotetext{
${ }^{1} \mathrm{CWI}$, Amsterdam, Netherlands.

${ }^{2}$ Instituto de Astrofísica de Andalucía, CSIC, Granada, Spain.

${ }^{3}$ Department of Physics, Eindhoven University of Technology, Eindhoven, Netherlands.
}

Copyright 2010 by the American Geophysical Union. 0094-8276/10/2009GL041982
[3] Many properties of sprites have recently been explained by adapting streamer models [Liu and Pasko, 2006; Liu et al., 2009a, 2009b]. These models, however, neglect the variation of air density and are therefore limited to sprite propagation over distances much smaller than the decaylength of the air density $(h=7.2 \mathrm{~km})$. Besides, they often use pointed metal electrodes to initiate the streamer discharge.

[4] We recently overcame these limitations [Luque and Ebert, 2009] by adaptive grid refinement and we showed how sprite streamers emerge from the collapse of a wide screening-ionization wave propagating downwards in the ionosphere. Here we apply similar techniques to the subsequent evolution of long sprite streamers. The results provide a natural explanation for the sprite phenomena discussed above.

\section{Model}

[5] In our previous work [Luque and Ebert, 2009] we investigated how a positive lightning stroke first generates a screening-ionization wave that can be visible as a halo [Barrington-Leigh et al., 2001], and how then the initial sprite streamer shoots out of the destabilizing wave (as observed by Cummer et al. [2006]). In this letter we investigate the next step of evolution: the propagation of a longer sprite after the initial wave stage. Therefore, we implement a late stage of wave evolution as initial condition here, namely the stage where a steep front between the interior of the ionospheric $\mathrm{E}$ region and the attachment hole below it already has formed. To compare with the observations of McHarg et al. [2007] and Stenbaek-Nielsen and McHarg [2008], we set the wave front at $85 \mathrm{~km}$ altitude and the ionization density above $85 \mathrm{~km}$ to 10 electron-ion pairs per $\mathrm{cm}^{3}$. The developing instability at the lower edge of the ionospheric wave is mimicked by a gaussian ionization seed of width $200 \mathrm{~m}$ at $85 \mathrm{~km}$ altitude and with a highest density of 90 electron-ion pairs per $\mathrm{cm}^{3}$. The initial ionization is electrically neutral everywhere.

[6] The simulated domain extends from 55 to $90 \mathrm{~km}$ in the vertical direction with radial extension of $25 \mathrm{~km}$; the finest grid resolution is $2.4 \mathrm{~m}$. Focusing on the propagating narrow sprite streamer, we ignore the spatial variation of the cloudgenerated electric field and set it to $40 \mathrm{~V} / \mathrm{m}$ everywhere.

[7] Otherwise our atmospheric discharge model is the same as in our previous work [Luque and Ebert, 2009]; it is described in detail in the supplementary material of that article. As electric conductivity is dominated by electron transport, we neglect ion mobility. Electrons drift in the local electric field, they are approximated as densities with altitude-dependent transport coefficients. Additional electron-ion-pairs are generated by impact ionization and photo-ionization, while electrons get lost mainly through 

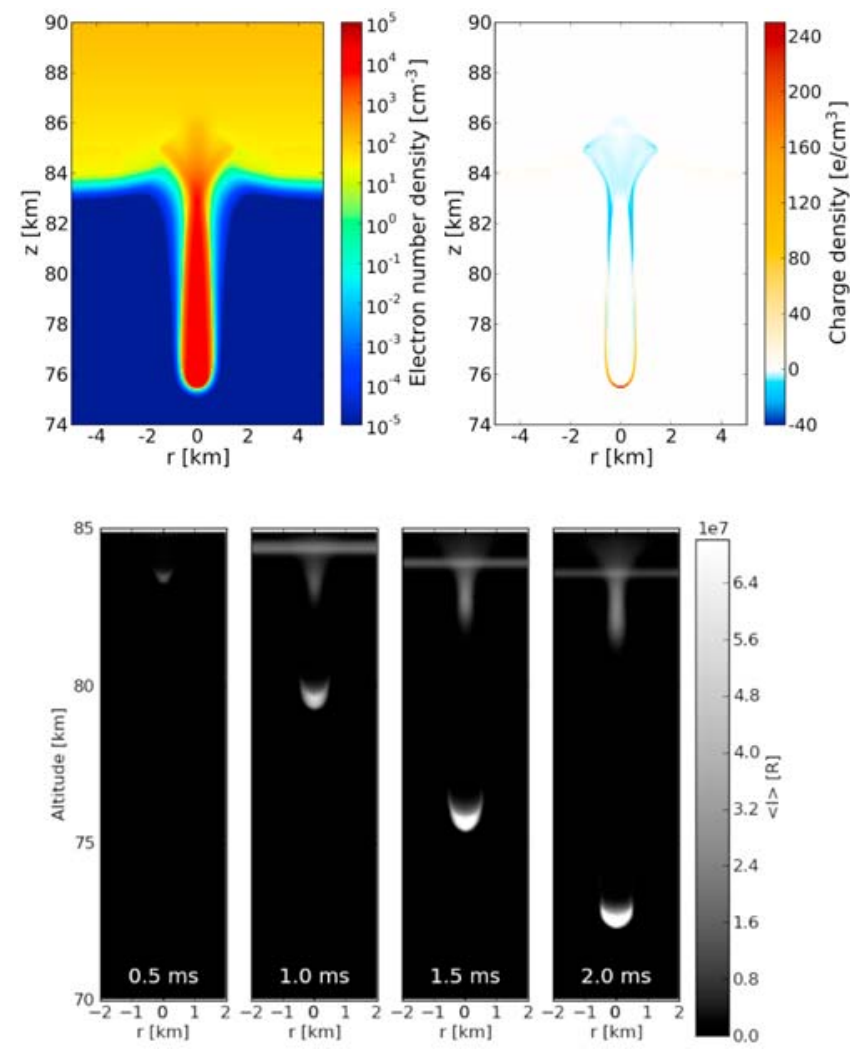

Figure 1. (top) (left) Electron number density (in $1 / \mathrm{cm}^{3}$ ) and (right) charge density (in e/ $\mathrm{cm}^{3}$, where e is the elementary charge) of the sprite streamer after $1.5 \mathrm{~ms}$ of evolution. Movies of the complete evolution and plots of the reduced electric field and the radial component of the electric field are available in the auxiliary material. ${ }^{1}$ (bottom) Optical emissions in the first positive band of $\mathrm{N}_{2}$ averaged over the preceding $50 \mu \mathrm{s}$. The emissions are integrated along a line of sight and are measured in Rayleigh (R), $\left(10^{10}\right.$ photons/ $\left.\mathrm{m}^{2} / \mathrm{s}\right)$. The scale is truncated to emphasize the trail emissions (the highest intensity reaches $2 \cdot 10^{8} \mathrm{R}$ ). Usually, observations are strongly over-exposed in the head region [see, e.g., Stenbaek-Nielsen et al., 2007]. The horizontal line at about $85 \mathrm{~km}$ is the lower boundary of the ionized region: emissions from that plane are very faint but yield high intensities when integrated along exact horizontal lines.

dissociative attachment. Electric fields are calculated in electrostatic approximation.

[8] Similar models were used in previous sprite studies [Liu and Pasko, 2004; Luque et al., 2008], but we incorporate the altitude dependent air density and the ionosphere with its limited conductivity rather than an electrode.

\section{Results and Interpretation}

\subsection{Properties of the Streamer Head}

[9] Figures 1 and 2 illustrate the main features of a positive streamer propagating in air of increasing density. The overall structure of the discharge is similar to that of a streamer in homogeneous air. But in our simulation, the

${ }^{1}$ Auxiliary materials are available in the HTML. doi:10.1029/ 2009GL041982. streamer propagates from 85 to $72 \mathrm{~km}$ altitude, where the air density $N(z)$ increases by a factor of 6 . Some salient features show the effect of the varying density, and do not agree with some simple minded applications of similarity laws.

[10] We recall that according to Townsend scaling [Ebert et al., 2006; Liu and Pasko, 2006], streamers in different air densities $N$ are related by approximate similarity laws. If the reduced electric field $E / E_{k}$ (where the breakdown value $E_{k}$ is proportional to $N$ ) at the streamer head stays the same during propagation, then the ionization density in the streamer head should increase like $N^{2}$. The streamer diameter as well as the width of the space charge layer around the head should decrease like $1 / N$.

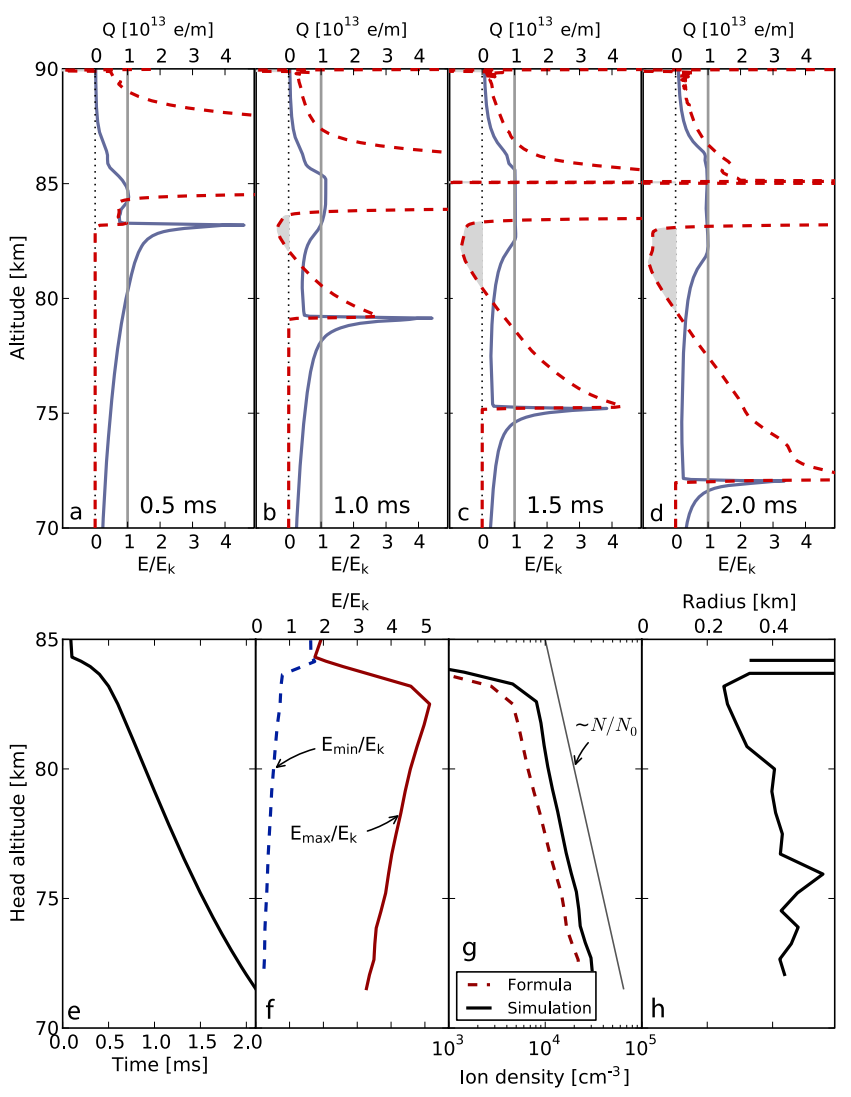

Figure 2. (a-d) Solid lines: reduced electric field $E / E_{k}$ on the symmetry axis at several time-steps. Dashed lines: Space charge density integrated over the radial cross section of the discharge channel. The integrated space charge shows a growing positive charge followed by a negatively charged region (shaded). The diverging positive space charge layer at higher altitudes is the screening layer at the lower edge of the ionosphere. The irregularities in the charge density at $2.0 \mathrm{~ms}$ mark the onset of numerical errors, so we stop our simulation there. (e) Streamer head position as a function of time. (f) Reduced maximal electric field at the tip $\left(E_{\max } / E_{k}\right)$ and reduced screened field just behind the front $\left(E_{\min } / E_{k}\right)$. (g) Ion density on the axis just behind the front taken from the simulation and from equation ionization with the electric fields in Figure 2b; for comparison, the slope for an ion density proportional to $N / N_{0}$ is also plotted. (h) Streamer radius calculated by fitting a sphere by least-squares. The oscillations appear because a coarse grid with resolution $\sim 40 \mathrm{~m}$ was used here. 
[11] In our simulations this does not occur, and this is not a numerical artifact due to insufficient numerical resolution. Our simulations (see Figure 2) show that instead of decreasing, the streamer radius grows from about $300 \mathrm{~m}$ to $500 \mathrm{~m}$. Both the maximal and the minimal $E / E_{k}$ decrease during propagation, and the ionization density in the head increases approximately like $N$, rather than like $N^{2}$.

[12] The consistency of these results can be tested by applying an estimate from the appendix of Li et al. [2007]. It relates the ionization density $n^{-}$behind a planar ionization front to the maximal electric field $E_{\max }$ ahead of the front and to the field dependent $\alpha(E)$ through

$$
n^{-}=\frac{\epsilon_{0}}{e} \int_{0}^{\left|E_{\max }\right|} \alpha(e) d e
$$

where $\epsilon_{0}$ is the dielectric constant, and $e$ is the elementary charge; here photo-ionization is neglected. The dashed line in Figure $2 \mathrm{~g}$ shows the ionization density predicted by ionization when the $E_{\max }$ from the simulation is inserted. The values lie at $\sim 2 / 3$ of the actual simulation values. If $E_{\max }$ would increase like $E_{k} \propto N$, the ionization density $n^{-}$would increase like $N^{2}$ according to this formula. But $E_{\max }$ increases less, and in such a manner that practically the ionization density increases like $N$. We have no explanation for this surprising fact yet. Another observation is that the minimal electric field $E_{\min }$ in the streamer head stays approximately constant.

\subsection{Negative Charging of the Streamer Trail}

[13] Figure 2 shows a region inside the channel where $E \approx$ $E_{k}$. Looking at Figure 1, we can identify this region with a negative charging of the streamer channel.

[14] As discussed in the previous section, the densities of charged particles increase like $N$ so an exponentially increasing number of electrons is liberated around the head. These electrons drift upwards, where the ion density is basically frozen (neglecting small variations due to attachment). Hence at some point the electron density surpasses the ion density and creates a net negative charge that is responsible for the increase in the electric field, eventually to values above $E_{k}$; then a second ionization wave sets in.

[15] Many observations show an increase of luminosity in the streamer channel after about $1 \mathrm{~ms}$. Some authors [Kanmae et al., 2007; Sentman et al., 2008] called this "afterglow" and attributed it to the release of energy stored during the passage of the head in the vibrational levels of $\mathrm{N}_{2}$. Our simulations point to a different mechanism: a secondary wave of impact ionization and excitation of $\mathrm{N}_{2}$ in electric fields $E \approx E_{k}$, in agreement with observations by Morrill et al. [2002]. This issue and the comparison with observed spectral features of sprites will be discussed in the next section.

[16] Other observations in high-speed sprite imaging also suggest a negative charging of the streamer channel. The first is the emergence of negative (upward-propagating) streamers, always reported to occur from a previous channel and some milliseconds after the passage of a positive streamer head. In some observations [Stanley et al., 1999; Cummer et al., 2006; Stenbaek-Nielsen and McHarg, 2008] the emergence of negative streamers coincides with the lower edge of the trailing emissions.
[17] Also, the attraction of downward-propagating sprite streamers to a neighboring channel has been reported in high-speed observations [Cummer et al., 2006; StenbaekNielsen and McHarg, 2008]. This appears to occur more frequently in sprites than in laboratory discharges [Nijdam et al., 2009]. A negative charging of the streamers explains the observations and also why, up to our knowledge, upward propagating streamers have not been observed to reattach to previous channels.

[18] This charging mechanism is present as long as the charge content of the streamer head increases fast enough. For sprites this increase is dominated by the exponential increase in air density, but the acceleration or expansion of the streamer will create similar effects even in air of constant density. In our view, this phenomenon has not been studied properly in the streamer literature, although it was visible already in the figures of Vitello et al. [1994].

\subsection{Light Emission From Streamer Head and Trail}

[19] It is now well established [Sentman et al., 1995; Pasko, 2007; Kanmae et al., 2007; Liu et al., 2009b] that optical emissions from sprites are dominated by the first positive band of nitrogen. Figure 1 shows the emissions from that band in our model streamer. They are calculated according to the radiation model developed by Pasko et al. [1997] and Liu and Pasko [2004]. The negatively charged region with $E \approx E_{k}$ discussed above is here seen as a glowing filament separated from the streamer head and slowly extending downwards. This pattern of optical emissions appears in observations from high-speed cameras (see, in particular, Stenbaek-Nielsen and McHarg [2008, Figure 5]). Previous work [Morrill et al., 1998; Bucsela et al., 2003; Kanmae et al., 2007] (see Sentman et al. [2008] for a recent discussion on this issue) proposed a two-stage chemical mechanism to explain light-emissions from low-field regions. However, detailed chemical studies [Sentman et al., 2008; Gordillo-Vázquez, 2008] where electric fields are modeled as a single pulse do not show a delayed increase in optical emissions.

[20] We explain the glowing trail by an increase of the reduced electric field. The mechanism of light emission is the same as in the streamer head: excitation of nitrogen by electron impact. This has also been very recently proposed by Liu [2010]. Morrill et al. [2002] measured the intensity ratio between the second positive band of $\mathrm{N}_{2}$ and the first negative band of $\mathrm{N}_{2}^{+}$integrated during the complete sprite evolution (about $10 \mathrm{~ms}$ ), suggesting that the emitting states were excited under $E \approx E_{k}$. On the other hand, Liu et al. [2009a] analyzed data from the ISUAL instrument in the FORMOSAT-2 satellite and concluded that in the early stages a sprite emits light mostly from regions where $E \approx$ $4 E_{k}$. Our simulations explain both observations: initially most light is emitted from the streamer head where $E \approx 4 E_{k}$ but as it propagates a larger proportion of light is emitted from the trail, where $E \approx E_{k}$.

[21] In our simulations the light emission from the streamer head increases strongly as it propagates; the intensity is approximately proportional to the air density, and hence it increases exponentially in time in agreement with Stenbaek-Nielsen et al. [2007], as long as the velocity does not vary too much. 


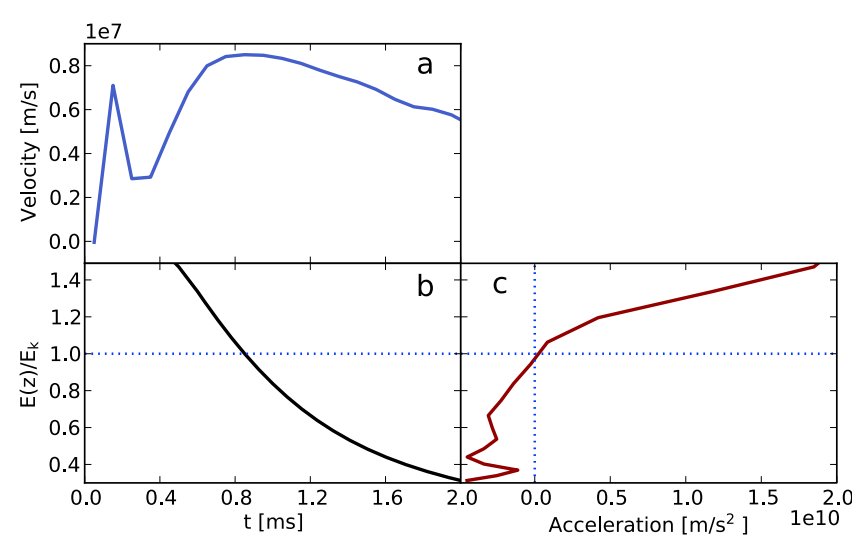

Figure 3. (a) Downward velocity as a function of time, (b) reduced electric field created by the cloud charge and the ionosphere at the location of the streamer head, and (c) relationship between acceleration and the reduced background electric field. Li and Cummer [2009] report a constant deceleration of about $10^{10} \mathrm{~m} / \mathrm{s}^{2}$ during the late stages of propagation; we observe similar values.

\subsection{Streamer Acceleration and Deceleration}

[22] We now focus on the streamer velocities. The streamer velocity depends significantly on the imposed electric field, so in our main run we selected an electric field to match the observed velocities [Li and Cummer, 2009; Cummer et al., 2006; Stenbaek-Nielsen and McHarg, 2008] of about $10^{7} \mathrm{~m} / \mathrm{s}$. Figure 3 a shows the velocity of the streamer head measured by the position of the highest positive space charge as a function of time. Within the first $0.6 \mathrm{~ms}$, the streamer accelerates up to a velocity of about $10^{7} \mathrm{~m} / \mathrm{s}$. Then it decelerates at a slower pace down to a velocity of about $6 \times 10^{6} \mathrm{~m} / \mathrm{s}$ at $1.5 \mathrm{~ms}$. A similar behavior was observed with high-speed cameras by Li and Cummer [2009].

[23] Figures $3 \mathrm{~b}$ and $3 \mathrm{c}$ shed some light on streamer acceleration and deceleration. Figure $3 \mathrm{~b}$ shows the reduced electric field at the altitude of the streamer head but far away in the radial direction $\left(E_{0}(z) / E_{k}\right)$. The streamer keeps accelerating in regions where $E_{0}(z)>E_{k}$ whereas it decelerates where $E_{0}(z)<E_{k}$. This is further illustrated by Figure $3 \mathrm{c}$, showing the acceleration of the streamer head.

[24] This picture suggests that sprite streamers are initiated in a region of super-critical field $E>E_{k}$. Then, they accelerate and propagate much faster than the screening wave, running into sub-critical regions where they decelerate at an almost constant rate.

\section{Summary and Conclusions}

[25] We have shown that the variation of air density along the propagation length of sprite streamers explains many of the observed and hitherto unexplained features of sprites. In addition, these results support our proposed mechanism of sprite inception [Luque and Ebert, 2009] and the relevance of a finite air conductivity in the region where sprite streamers are initiated. Many of our conclusions are based on phenomenological observations in our simulations. Future studies have to address the physical mechanism behind these phenomena.
[26] Acknowledgments. AL acknowledges a visit to CWI paid by the Dutch STW-project 10118 and partial support of MICINN under project AYA2009-14027-C05-02.

\section{References}

Barrington-Leigh, C. P., U. S. Inan, and M. Stanley (2001), Identification of sprites and elves with intensified video and broadband array photometry, J. Geophys. Res., 106, 1741-1750.

Bucsela, E., J. Morrill, M. Heavner, C. Siefring, S. Berg, D. Hampton, D. Moudry, E. Wescott, and D. Sentman (2003), $\mathrm{N}_{2}\left(\mathrm{~B}^{3} \Pi_{g}\right)$ and $\mathrm{N}_{2}^{+}\left(\mathrm{A}^{2} \Pi_{u}\right)$ vibrational distributions observed in sprites, J. Atmos. Sol. Terr. Phys., 65, 583-590.

Cummer, S. A., N. Jaugey, J. Li, W. A. Lyons, T. E. Nelson, and E. A. Gerken (2006), Submillisecond imaging of sprite development and structure, Geophys. Res. Lett., 33, L04104, doi:10.1029/2005GL024969.

Ebert, U., C. Montijn, T. M. P. Briels, W. Hundsdorfer, B. Meulenbroek, A. Rocco, and E. M. van Veldhuizen (2006), The multiscale nature of streamers, Plasma Sources Sci. Technol., 15, S118-S129.

Gerken, E. A., U. S. Inan, and C. P. Barrington-Leigh (2000), Telescopic imaging of sprites, Geophys. Res. Lett., 27, 2637-2640.

Gordillo-Vázquez, F. J. (2008), Air plasma kinetics under the influence of sprites, J. Phys. D Appl. Phys., 41, 234016.

Kanmae, T., H. C. Stenbaek-Nielsen, and M. G. McHarg (2007), Altitude resolved sprite spectra with $3 \mathrm{~ms}$ temporal resolution, Geophys. Res. Lett., 34, L07810, doi:10.1029/2006GL028608.

Li, C., W. J. M. Brok, U. Ebert, and J. J. A. M. van der Mullen (2007), Deviations from the local field approximation in negative streamer heads, J. Appl. Phys., 101, 123305.

Li, J., and S. A. Cummer (2009), Measurement of sprite streamer acceleration and deceleration, Geophys. Res. Lett., 36, L10812, doi:10.1029/ 2009GL037581.

Liu, N. (2010), Model of sprite luminous trail caused by increasing streamer current, Geophys. Res. Lett., 37, L04102, doi:10.1029/ 2009 GL042214.

Liu, N., and V. P. Pasko (2004), Effects of photoionization on propagation and branching of positive and negative streamers in sprites, J. Geophys. Res., 109, A04301, doi:10.1029/2003JA010064.

Liu, N., and V. P. Pasko (2006), Effects of photoionization on similarity properties of streamers at various pressures in air, J. Phys. D Appl. Phys., 39, 327-334

Liu, N., V. P. Pasko, H. U. Frey, S. B. Mende, H. Su, A. B. Chen, R. Hsu, and L. Lee (2009a), Assessment of sprite initiating electric fields and quenching altitude of $\mathrm{a}^{1} \Pi_{g}$ state of $\mathrm{N}_{2}$ using sprite streamer modeling and ISUAL spectrophotometric measurements, J. Geophys. Res., 114, A00E02, doi:10.1029/2008JA013735.

Liu, N. Y., V. P. Pasko, K. Adams, H. C. Stenbaek-Nielsen, and M. G. McHarg (2009b), Comparison of acceleration, expansion, and brightness of sprite streamers obtained from modeling and high-speed video observations, J. Geophys. Res., 114, A00E03, doi:10.1029/2008JA013720.

Luque, A., and U. Ebert (2009), Emergence of sprite streamers from screening-ionization waves in the lower ionosphere, Nat. Geosci., 2, $757-760$.

Luque, A., U. Ebert, and W. Hundsdorfer (2008), Interaction of streamer discharges in air and other oxygen-nitrogen mixtures, Phys. Rev. Lett., 101, 075005 .

McHarg, M. G., H. C. Stenbaek-Nielsen, and T. Kammae (2007), Observations of streamer formation in sprites, Geophys. Res. Lett., 34, L06804, doi:10.1029/2006GL027854.

Morrill, J., et al. (2002), Electron energy and electric field estimates in sprites derived from ionized and neutral $\mathrm{N}_{2}$ emissions, Geophys. Res Lett., 29(10), 1462, doi:10.1029/2001GL014018.

Morrill, J. S., E. J. Bucsela, V. P. Pasko, S. L. Berg, M. J. Heavner, D. R. Moudry, W. M. Benesch, E. M. Wescott, and D. D. Sentman (1998), Time resolved $\mathrm{N}_{2}$ triplet state vibrational populations and emissions associated with red sprites, J. Atmos. Sol. Terr. Phys., 60, 811-829.

Nijdam, S., C. G. C. Geurts, E. M. van Veldhuizen, and U. Ebert (2009), Reconnection and merging of positive streamers in air, J. Phys. D Appl. Phys., 42, 045201.

Pasko, V. P. (2007), Red sprite discharges in the atmosphere at high altitude: The molecular physics and the similarity with laboratory discharges, Plasma Sources Sci. Technol., 16, S13-S29.

Pasko, V. P., U. S. Inan, T. F. Bell, and Y. N. Taranenko (1997), Sprites produced by quasi-electrostatic heating and ionization in the lower ionosphere, J. Geophys. Res., 102, 4529-4561.

Sentman, D. D., E. M. Wescott, D. L. Osborne, D. L. Hampton, and M. J. Heavner (1995), Preliminary results from the Sprites94 aircraft campaign: 1. Red sprites, Geophys. Res. Lett., 22, 1205-1208.

Sentman, D. D., H. C. Stenbaek-Nielsen, M. G. McHarg, and J. S. Morrill (2008), Plasma chemistry of sprite streamers, J. Geophys. Res., 113, D11112, doi:10.1029/2007JD008941. 
Stanley, M., P. Krehbiel, M. Brook, C. Moore, W. Rison, and B. Abrahams (1999), High speed video of initial sprite development, Geophys. Res. Lett., 26, 3201-3204.

Stenbaek-Nielsen, H. C., and M. G. McHarg (2008), High time-resolution sprite imaging: Observations and implications, J. Phys. D Appl. Phys., $41,234009$.

Stenbaek-Nielsen, H. C., M. G. McHarg, T. Kanmae, and D. D. Sentman (2007), Observed emission rates in sprite streamer heads, Geophys. Res. Lett., 34, L11105, doi:10.1029/2007GL029881.
Vitello, P. A., B. M. Penetrante, and J. N. Bardsley (1994), Simulation of negative-streamer dynamics in nitrogen, Phys. Rev. E, 49, 5574-5598.

U. Ebert, CWI, PO Box 94079, NL-1090 GB Amsterdam, Netherlands. (ebert@cwi.nl)

A. Luque, Instituto de Astrofísica de Andalucía, CSIC, PO Box 3004, E-18080 Granada, Spain. (aluque@iaa.es) 